\title{
Comparison of Placental Histopathology in IUG Rand Normal Term Infants: A Cross-Sectional Study
}

\author{
SuneetaSingh ${ }^{\mathrm{a}} \mathrm{MS}, \mathrm{DNB}$, Arpitha Pemmaraju ${ }^{\mathrm{b}} \mathrm{MD}$, DevendraNema ${ }^{\mathrm{c}} \mathrm{MD}$ \\ ${ }^{A}$ classified Specialist (Obstetrics \& Gynaecology), 155 Base Hospital, C/O 99 APO \\ ${ }^{B}$ Classified Specialist (Pathology), 155 Base Hospital, C/O 99 APO \\ ${ }^{C}$ Asst Professor (Radiology), Command Hospital, Lucknow (UP)
}

\begin{abstract}
:
Introduction: Healthy development of fetus is dependent on normal placental development. Alteration in the placental development is the main etiopathological factor for IUGR. The present study aims to compare the histopathological findings of placenta in IUGR and normal term delivery.

Materials and Methods: This cross sectional study was done at an Army zonal Hospital from Jan 2016 to Jan 2017. Sixty placenta were studied of which 30 belonged to the IUGR group and other 30 were from normal term fetus( Control group). Inclusion criteria was maternal age between 20 to 35 yrs, Gestational age between 32 to 40.5 wks with IUGR(USG confirmed) or normal healthy fetus. Exclusion criteria were twin pregnancy, gestational age $<32 w k s$, and diabetes. Data of mother, newborn and placenta were recorded after delivery. The subjects were matched for age, height, weight, social status. Data were analyzed using SPSS software version 16.

Results: Infarction rate, thrombosis, tissue ischemia, intervillous haemorrhage and intervillous fibrin deposition were significantly higher in IUGR group. Mean placenta weight was lower in IUGR group (380 gms vs. 510 gms).

Conclusion:Placenta of IUGR newbornswere smaller with more microscopic infarction. The findings suggest that chronic ischemia and associated secondary changes probably lead to improper perfusion and IUGR. The gross and microscopic measurements of a placenta are more objective and seem to offer a good way to get proper information about IUGR. Therefore a detailed history of placental study should be taken during ANC visit of a lady with previous history of IUGR and manage the case accordingly. All IUGR fetuses must be considered at risk for poor placental circulation and hypoxia along with compromised nutrition. This study therefore indicates that study of fetal blood supply must be done as a part of surveillance of IUGR cases by Doppler study and other tests of fetal well-being must be carried out during antenatal period frequently and they must be delivered as soon as the fetal maturity is reasonably assured or there are any signs of fetal compromise.
\end{abstract}

Keywords:IUGR, Placenta, Calcification, Thrombosis, infarction, intervillous fibrin

\section{Introduction}

Intra uterine growth restriction (IUGR) is most commonly defined as weight of fetus less than tenth centile for that gestational age ${ }^{1}$. Intra uterine growth restriction is a common clinical sign for chronic fetal hypoxemia. FGR is one of the leading causes of perinatal morbidity and mortality ${ }^{2,3}$. Placenta is a vital organ for normal development of a healthy fetus as it is the media of transfer of essential nutrients and oxygen from mother to fetus and the waste products of metabolism from fetus to the mother. Any aberration in its development may have catastrophic effect on both the fetus and mother in the form of IUGR, IUFD and preeclampsia, Abruptio placenta respectively.A placenta is like a diary of gestational life ${ }^{4}$. It should be studied carefully. The etiology of IUGR can be divided into maternal, fetal, placental and unknown causes but placenta holds the main role in the basic pathophysiology of IUGR ${ }^{5}$.Impaired placentation is thought to underlie most cases of IUGR especially early IUGR and early onset pre-eclampsia which is characterised by placental pathology associated with hypoxia and reperfusion damage caused by impaired remodelling of spiral arteries ${ }^{3,6}$.

In a histopathological evaluation of placenta in IUGR pregnancies, the weight of IUGR placenta was less than normal placenta. Infarction and intervillousfibrinoid deposition were higher in IUGR placenta. In addition thickening of basal membrane and cytotrophoblast hyperplasia were more common among IUGR placenta. All the main histopathological findings pointed to placental blood flow reduction and fetal blood flow restriction ${ }^{7}$

The present study was conducted to compare the pathological changes in IUGR with placental histology in term normal infants so as to establish a relationship between placental pathology and IUGR. This study may be useful in deciding and managing future pregnancies. Prevention of the perinatal complications in fetuses with growth restriction is possible by effective antenatal identification and management. 


\section{Materials \& Methods}

This cross sectional study was done at an Army zonal Hospital from Jan 2016 to Jan 2017. Sixty placenta were studied of which 30 belonged to the IUGR group and other 30 were from normal term fetus (Control group). The day a sample of placenta of IUGR case was sent, a placenta from normal full term delivery without any associated comorbidity was sent for gross and HPE .

Inclusion criteria was maternal age between 20 to $35 \mathrm{yrs}$, Gestational age between 32 to 40.5 wks with IUGR(USG confirmed) or normal healthy fetus.

IUGR was clinically suspected and then confirmed by USG showing growth lag of more than 3 Wks .These cases were further followed by regular close fetal surveillance in the form of Daily fetal movement count, Non stress test and umbilical flow velocimetry studies and USG for interval growth. Surfactant induction (Inj Betamethasone $12 \mathrm{mg}$ IM 24 hrly 2 doses) was given once IUGR was confirmed.IUGR cases weredelivered as soon as the fetal maturity was reasonably assured or there are any signs of fetal compromise.

Exclusion criteria were Twin pregnancy, GA $<32$ wks, GDM.

A thorough history, general and systemic examination of patient was done.

Placenta obtained after delivery was sent immediately in $10 \%$ formalin to the lab for gross and histopathological examination by pathologist. Gross examination was carried out on the same day on intact specimen and after bread- loafing. The following parameters were noted:

- Cord insertion -central or eccentric

- Number of vessels in the cord

- Membrane insertion - marginal or circumvallate

- Membrane discolouration - meconium stained or yellow

- Areas of infarction, calcification, fibrin deposition.

Sections are taken from cord, membranes and parenchyma. Sections from membranes include the rupture site. These are rolled and processed further. The sections from parenchyma include all relevant pathological areas and atleast one "normal" area. These are full thickness sections including the chorionic plate. The sections are further fixed overnight in $10 \%$ formalin and processed. Routine Hematoxylin and Eosin stains are employed for all sections.

The results obtained were analysed to study the correlation between placental findings and the delivery outcomes. Statistical correlation was done using SPSS software version 16.

$\mathrm{P}<0.05$ was taken as critical level of significance.

\section{Results}

Maternal characteristics of the study group and the control group is shown in Table 1.

Table 1

\begin{tabular}{|l|l|l|}
\hline & Study Group (30) & Control Group (30) \\
\hline Mean Age & 26.6 Yrs & $26.4 \mathrm{Yrs}$ \\
\hline Mean Wt & $64.3 \mathrm{~kg}$ & $66.9 \mathrm{~kg}$ \\
\hline Mean Gestational Age & $36.95 \mathrm{Wks}$ & $38.7 \mathrm{Wks}$ \\
\hline Significant Contributory Finding & $\begin{array}{l}3 \mathrm{PIH}, 2 \text { APLA positive, } \\
1 \text { Thrombophilia }\end{array}$ & - \\
\hline Average Birth Weight & $2.24 \mathrm{~kg}$ & $3.01 \mathrm{~kg}$ \\
\hline
\end{tabular}

The gross findings of placenta of the study group and the control group is shown in Table 2:

Table 2

\begin{tabular}{|l|l|l|}
\hline & Study Group (30) & Control Group (30) \\
\hline Average Placental weight & 380 gms & 510 gms \\
\hline Cord insertion & $\begin{array}{l}\text { Central - 11 } \\
\text { Eccenteric - 19 }\end{array}$ & $\begin{array}{l}\text { Central - 19 } \\
\text { Eccenteric - 11 }\end{array}$ \\
\hline Membrane discolouration & $\begin{array}{l}\text { Green - 5 } \\
\text { Yellow - 1 }\end{array}$ & $\begin{array}{l}\text { Green - 1 } \\
\text { Yellow - 0 }\end{array}$ \\
\hline Membrane insertion & $\begin{array}{l}\text { Marginal - 29 } \\
\text { Circumarginate- 2 }\end{array}$ & $\begin{array}{l}\text { Marginal - 30 } \\
\text { Circumarginate- 0 }\end{array}$ \\
\hline Gross infarction & 6 & 2 \\
\hline Calcification & 1 & 0 \\
\hline
\end{tabular}


Comparison Of Placental Histopathology In IUGR And Normal Term Infants: A Cross-Sectional Study...

The microscopic findings of placenta of the study group and the control group is shown in Table 3:

Table 3

\begin{tabular}{|l|l|l|l|}
\hline & Study Group (30) & Control Group (30) & P value \\
\hline Placental infarction & 19 & 02 & $<0.001$ \\
\hline Ischemic changes & 26 & 04 & $<0.001$ \\
\hline Thrombosis & 15 & 02 & $<0.01$ \\
\hline Calcification & 14 & 04 & $<0.001$ \\
\hline Chorangiosis & 03 & 0 & Not significant \\
\hline $\begin{array}{l}\text { Intervillousfibrinoid } \\
\text { deposition }\end{array}$ & 26 & 05 & $<0.001$ \\
\hline Cord vasculitis & 0 & 0 & Not significant \\
\hline Choroioamnionitis & 3 & 0 & Not significant \\
\hline Intervillous hemorrhage & 25 & 0 & $<0.001$ \\
\hline
\end{tabular}

Grading of intervillousfibrinoid deposition seen in the study group is shown in Table 4:

Table 4

\begin{tabular}{|l|l|l|}
\hline Intervillousfibrinoid deposition & Study Group (30) & Control Group (30) \\
\hline$<5 \%$ & 8 & 3 \\
\hline $5 \%-10 \%$ & 8 & 2 \\
\hline$>10 \%$ & 10 & 0 \\
\hline
\end{tabular}

Fig 1 A- F are showing the pathological findings in IUGR placenta

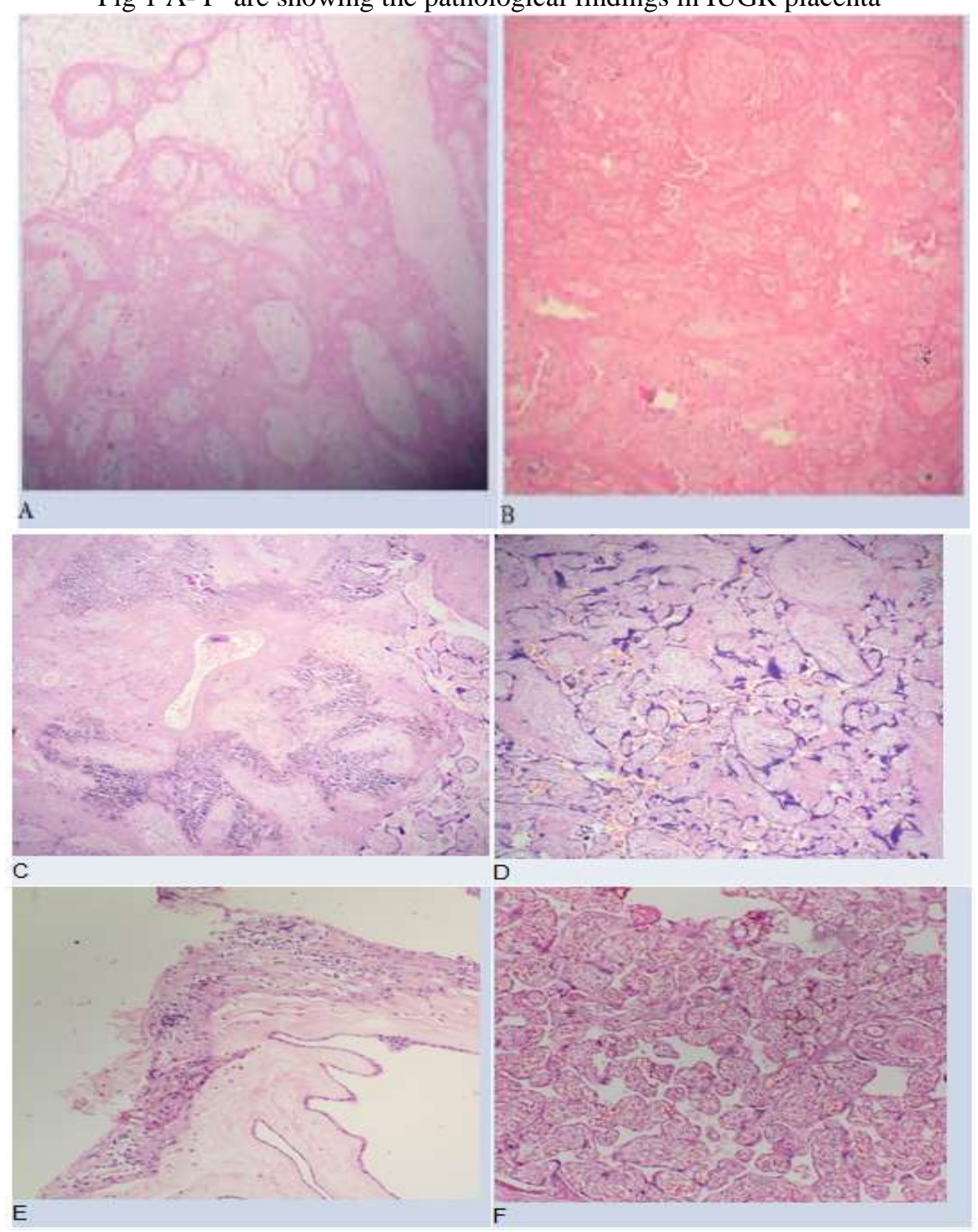

Fig 1A,B- Placental infarct . Fig 1C - Calcification with intervillousfibrinoid deposition.

Fig 1D - Increased syncytial knots and intervillousfibrinoid deposition.

Fig 1E -Chorioamnionitis.Fig 1F - Chorangiosis 
Fig 2 A,B are showing the microscopic appearance of normal placenta from a normal term delivery

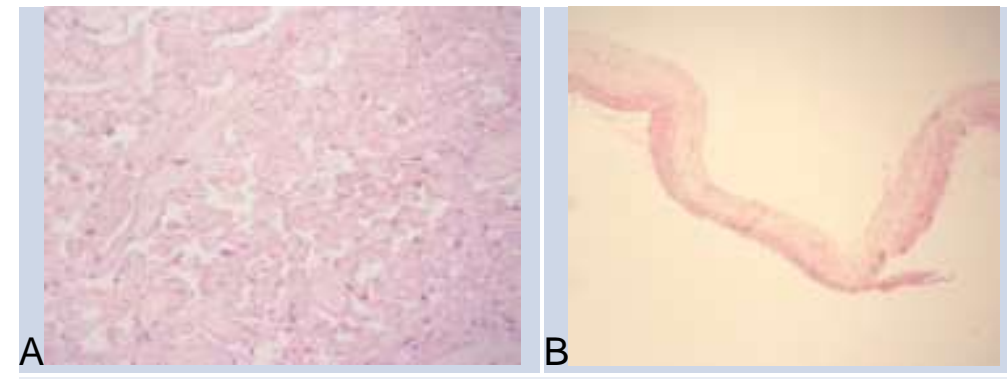

Fig 2A - Normal Mature Placenta .Fig 2 B -Section from membranes of mature Placenta

\section{Discussion}

Chronic placental insufficiency accounts for majority of IUGR foetuses. In this study we have tried to study the significant placental pathology in IUGR cases.Mean Placental weight of IUGR cases was $380 \mathrm{gm}$ (less than $25^{\text {th }}$ percentile $)$ as compared to the $510 \mathrm{gm}\left(>50^{\text {th }}\right.$ percentile $)$ in control group. Similar finding was seen in other studies ${ }^{8,9}$.Few studies suggested circumvallate placenta, circummarginate, velamentous insertion of the cord and placenta previa as possible causes of $\operatorname{IUGR}^{1,10,11}$. In this study almost all the placenta had marginal insertion of membranes. No significance can hence be attached to this finding in our study.

IUGR placenta generally shows pathological lesions of two major categories ${ }^{12}$ :

i. Due to reduced vascular supply of nutrients and oxygen (eg: infarction, villous hypoplasia, increased syncytial knotting

ii. Due to altered inflammatory response (eg: chorioamnionitis, villitis )

In our study we found signs of chronic placental insufficiency in the form of ischemia, intervilloushaemorrhages, calcification, fibrinoid necrosis, intravillous fibrin deposition in IUGR group placenta in significant number of cases. The $p$ value was $<0.01$ for all the above mentioned features as compared to the control group. The same has been quoted in previous studies ${ }^{9,13-17}$. In this study the inflammatory response was seen in only 2 of the 30 cases of IUGR. Redline and Pappin have suggested the association between chronic villitis and growth restriction ${ }^{19}$. Veerbbeketaland Green LG etalalso observed higher level of chronic chorioamnionitisfrom pregnancies with early IUGR and maternal hypertensive disease as compared to normotensive IUGR pregnancies and suggested that pronounce allograft rejection and graft versus host disease in membranes in women with concomitant hypertensive disease ${ }^{14,20}$. This could be due to the small sample size combined with only three cases of PIH in our study. Therefore, it is recommended such pathological findings be examined in a larger sample size in future studiesIntervillousfibrinoid deposition of more than $10 \%$ was more common in the study group as was seen in previous studies ${ }^{9,13,15,16}$. Maternal thrombophilias, both inherited and acquired have been seen to be associated with increased fibrinoid deposition in intervillous space. This results in trapping of villi by the fibrinoid material and ultimately necrosis. Therefore, high amount of intervillousfibrinoid deposition is a pathological finding in IUGR-related placenta ${ }^{22}$.

Chen etal studied the relationship between preterm placental calcification and adverse pregnancy outcome by ultrasonography and they concluded that early preterm placental calcification is associated with a higher incidence of adverse pregnancy outcome and may serve as an indicator of adverse maternal and fetal outcomes when noted on ultrasonography ${ }^{21}$. Conversely, women with late preterm placental calcification are not at greater risk for adverse pregnancy outcome. In the present study we observed calcification in $46.6 \%$ cases $(14 / 30)$ of IUGR placentae as compared with $13.33 \%(4 / 30)$.

\section{Conclusion}

Placenta of IUGR newborns was smaller with more gross finding of calcification and more microscopic infarction. Significant pathologic findings were infarction, thrombosis, ischemia, intervillushaemorrhage and intervillus fibrosis. These findings suggest that chronic ischemia and associated secondary changes probably lead to improper perfusion and IUGR. Therefore all pregnancies diagnosed to have IUGR antenatally must be suspected to have etiology of placental origin unless proved otherwise. The corollary to this is that such IUGR fetuses must be considered at risk for poor placental circulation and hypoxia along with compromised nutrition. This study therefore indicates that study of fetal blood supply must be done as a part of surveillance of IUGR cases by Doppler study and other tests of fetal well-being must be carried out during antenatal period frequently and they must be delivered as soon as the fetal maturity is reasonably assured or there are any signs of fetal compromise. 


\section{References}

[1]. Maulik D, Frances Evans J, Ragolia L. Fetal growth restriction: pathogenic mechanisms. ClinObstetGynecol 2006;49(2):219-27.

[2]. Lawn JE, Blencowe H, Pattinson R etal. Stillbirths: Where?When? Why? How to make the data count? Lancet 2011 Apr 23; 377(9775): 446-63.

[3]. Brossens I, Pijnenborg R, Vercruysse L, Roero R. The " Great Obstetrical Syndromes" are associated with disorders of deep placentation. Am J ObstetGynecol 2011 Mar; 204 (3): 193- 201

[4]. Geoffrey P Altshuler Chapter 50, 2301. Sterberg's Diagnostic Surgical Pathology. $4^{\text {th }}$ edn

[5]. Dutta DC. 7th ed. Kolkata: New Central Book Agency (P) Ltd; 2010. Text Book of Obstetrics Including Perinatology and Contraception; pp. 456-67.

[6]. Kovo M, Schreiber L, Bar J. Placental vascular pathology as mechanism of disease in pregnancy complications. Thromb Res 2013 Jan; 131 ( Suppl. 1): S 18-21

[7]. Curtin WM, Krauss S, Metlay LA, Katzman PJ. Pathologic examination of the placenta and observed practice. ObstetGynecol 2007J;109(1):35-41.

[8]. Roberts DJ, Post MD. The placenta in pre-eclampsia and intrauterine growth restriction. J ClinPathol. 2008;61:1254-60. [PubMed]

[9]. JS Nigam, V Misra, P SinghHistopathological Study of Placentae in Low Birth Weight Babies inIndia.Ann Med Health Sci Res.2014 Jul-Aug; 4(Suppl 2): S79-S83.

[10]. BenirschkeK, Kaufmann P, Baergen R. Pathology of the Human Placenta. 5th ed. New York Springer 2006. 9. Faye-Petersen OM, Heller DS, Joshi VV. Handbook of Placental Pathology. 2nd ed. London:Taylor\& Francis;2006.

[11]. Salafia CM, Charles AK, Maas EM. Placenta and fetal growth restriction. ClinObstetGynecol 2006;49(2):236-56.

[12]. Bernstein IM, Horbar JD, Badger GJ, Ohlsson A, Golan A. Morbidity and mortality among very low birth weight neonates with intra uterine growth restriction. The Vermont Oxford Network. Am J ObstetGynecol 2000 Jan ; 182 (1 Pt 1): 198-206

[13]. Mardi K, Sharma J. Histopathological evaluation of placentas in IUGR pregnancies. Indian J PatholMicrobiol 2003;46(4):551-4

[14]. JHW Veerbeek, P G J Nikkels, H L Torrance etal . Placental Pathology in Early Intrauteine Growth Restriction Associated with Maternal Hypertension. Placenta 35 (2014); 696-701

[15]. NayerehGhomian, SakinehAmouian, FatemehTavassoli, Taran. Comparison of Placental Morphology and Histopathology of Intrauterine Growth Restriction and Normal Infants. Iranian Journal of Pathology (2014) 9 (1), 9- 16.

[16]. VarunShirishkar, KiranPatole. Study of Placental Insufficiencies in IUGR Babies in Term Pregnancies. MVP Journal of Medical Sciences, Vol 1 (2); 80-83, Jul 2014

[17]. Kleebkaow P, Limdumrongchit W, Ratanasiri T, Komwilaisak R, Seejorn K. Prevalence of placental pathology in low birthweight infants. J Med Assoc Thai. 2006;89:594-9. [PubMed]

[18]. Redline RW, Boyd T, Campbell V, Hyde S, Kaplan C, Khong TY, et al. Maternal vascular underperfusion: Nosology and reproducibility of placental reaction patterns. PediatrDevPathol. 2004;7:237-49

[19]. Redline RW. Villitis of unknown etiology: Noninfectious chronic villitis in the placenta. Hum Pathol. 2007;38:1439-46. [PubMed]

[20]. Greer LG, Ziade MS, Casey BM etal. An immunologic basis for Placental insufficiency in fetal growth restriction. Am J Perinatol 2012 Aug; 29 (7): 533-8

[21]. Chen KH, Chen LR, Lee YH. Exploring the relationship between preterm placental calcification and adverse maternal and fetal outcome. Ultrasound Obstet Gynecol. 2011;37:328-34. [PubMed]

[22]. Drucilla J Roberts. Placental Pathology, a survival guide. Arch Pathol Lab Med, Vol 132; 641-51, Apr 2008. 